\title{
Anisotropic magnetoresistance in nanocontacts
}

\author{
D. Jacob, ${ }^{1,2}$ J. Fernández-Rossier, ${ }^{2}$ and J. J. Palacios ${ }^{2}$ \\ ${ }^{1}$ Department of Physics and Astronomy, Rutgers University, Piscataway, New Jersey 08904, USA \\ ${ }^{2}$ Departamento de Física Aplicada, Universidad de Alicante, San Vicente del Raspeig, 03690 Alicante, Spain
}

(Received 9 August 2007; revised manuscript received 3 January 2008; published 10 April 2008)

\begin{abstract}
We present $a b$ initio calculations of the evolution of anisotropic magnetoresistance (AMR) in Ni nanocontacts from the ballistic to the tunnel regime. We find an extraordinary enhancement of AMR, compared to bulk, in two scenarios. In systems without localized states, such as chemically pure break junctions, large AMR only occurs if the orbital polarization of the current is large, regardless of the anisotropy of the density of states. In systems that display localized states close to the Fermi energy, such as a single electron transistor with ferromagnetic electrodes, large AMR is related to the variation of the Fermi energy as a function of the magnetization direction.
\end{abstract}

DOI: 10.1103/PhysRevB.77.165412

PACS number(s): 75.47.- m, 72.15.Gd, 73.63.Rt

\section{INTRODUCTION}

The free energy of monodomain ferromagnetic particles depends on the relative orientation of the magnetization with respect to the crystal lattice. This magnetic anisotropy results from the combination of the Coulomb repulsion favoring spin polarization, spin-orbit coupling (SOC), and the crystal field breaking the orbital rotation invariance. As a result, the orbital moment of magnetic atoms and their magnetic anisotropy energy (MAE) depend strongly on their atomic coordination. ${ }^{1,2}$

The transport counterpart of MAE is anisotropic magnetoresistance (AMR), i.e., the dependence of the resistance on the angle $\theta$ between the magnetization and the current flow. Whereas AMR in bulk was known back in the 19th century and is a rather small effect, the recent observation of AMR in a variety of low dimensional systems, ${ }^{3-12}$ largely exceeding bulk values, has opened a new research venue in the field of spin-polarized quantum transport. Very large AMR has been reported in planar tunnel junctions [tunneling anisotropic magnetoresistance (TAMR)] with a variety of electrode and barrier materials. ${ }^{3-8}$ Enhanced AMR has also been observed in atomic sized contacts, both in the tunnel regime (TAMR) and in the contact (or ballistic ${ }^{13}$ ) regime [ballistic anisotropic magnetoresistance (BAMR)], ${ }^{14}$ for Py, ${ }^{9} \mathrm{Fe},{ }^{10} \mathrm{Ni},{ }^{11}$ and Co. ${ }^{12}$ Additionally, GaMnAs islands in the Coulomb blockade regime show electrically tunable AMR. ${ }^{15}$ Thus, a wide range of nanostructures made from different materials display enhanced AMR.

Here, we focus on AMR in atomic-sized conductors for several reasons. On the one hand, conductance of atomicsized contacts probes the electronic structure of the apex atoms. These have coordination different from bulk and thus present different orbital and spin magnetic moment ${ }^{16}$ and enhanced magnetic anisotropy ${ }^{1,2,17}$ which might be probed by BAMR. On the other hand, nanocontacts allow us to study AMR going from the contact (BAMR) to the tunnel (TAMR) regime in the same system, as shown in the case of both $\mathrm{Ni}$ and $\mathrm{Py} .{ }^{9,18} \mathrm{Ni}$ nanocontacts have also been used as electrodes to explore the Coulomb blockade and the Kondo regimes. ${ }^{19}$

The crux of the matter is to identify the necessary and sufficient conditions to expect large values of AMR in quan- tum transport. Here, we consider two different transport regimes: coherent and sequential. In the coherent regime, we use the Landauer formalism that, at zero temperature, relates the zero-bias conductance $G$ to the quantum mechanical transmission of the electrons at the Fermi energy, $G$ $=\frac{e^{2}}{h} T\left(\epsilon_{F}\right)$. This approach accounts for AMR both in the tunneling regime (TAMR) ${ }^{20}$ and in the contact or ballistic regime (BAMR) $)^{14}$ in the absence of sharp resonances near the Fermi energy. In the scattering-free case of perfect onedimensional (1D) chains, $T\left(\epsilon_{F}\right)$ is simply given by the number of bands at the Fermi energy $\mathcal{N}\left(\epsilon_{F}\right)$. Because of the SOC, $\mathcal{N}\left(\epsilon_{F}\right)$ for ferromagnetic 1D transition metal chains ${ }^{10,12,14,17}$ depends on the angle $\theta$ between the chain axis and the magnetization, and this leads naturally to stepwise $G(\theta)$ curves.

However, the idealized scattering-free picture fails to account for the experimental results of conductance in metallic nanocontacts, for which scattering channels are not perfectly conducting. ${ }^{21}$ According to the scattering-free theory, the conductance of atomic contacts of $\mathrm{Ni}$, in units of $e^{2} / h$, would be 6 or 7 for $\mathrm{Ni},{ }^{14}$ in quantitative disagreement with the measured ${ }^{22}$ conductance of Ni nanocontacts around $3 e^{2} / h$. The same applies to $\mathrm{Fe}, \mathrm{Co}$, and Pt. Scattering definitely affects $d$ bands, which suffer the so-called orbital blocking. ${ }^{23}$

Here, we present calculations of BAMR plus scattering. This approach also permits us to calculate the crossover from BAMR to TAMR. We find that in the coherent regime, large AMR is related to the orbital polarization of the current. TAMR has been linked to the anisotropy of the density of states at $\epsilon_{F}$, which turns out to be large in Ni chains. Unexpectedly, this does not lead to a large value of TAMR, the reason being that the current is not orbitally polarized in this limit.

In the sequential regime, which is valid to describe systems that feature transport through resonant levels of width $\Gamma$ smaller than the temperature $k T,{ }^{24}$ we find enhanced AMR, regardless of the orbital polarization of the current, if the chemical potential $\epsilon_{F}$ of the ferromagnetic electrode crosses a resonance as it varies due to change of the magnetization angle. This situation occurs in a single electron transistor with ferromagnetic electrodes. ${ }^{15,25}$ Resonances might also occur in the tip atoms of $\mathrm{Ni}$ nanocontacts in the tunneling regime. ${ }^{26}$ 


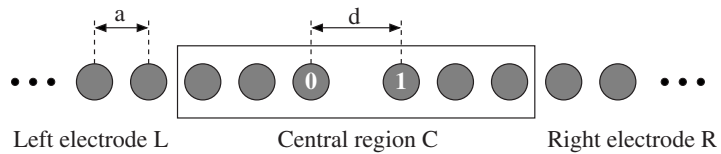

FIG. 1. 1D model of Ni nanocontact: elastic electron scattering in the contact region of a real nanocontact is mimicked by a gap $d>a$ between two semi-infinite 1D Ni chains with lattice spacing $a$.

This paper is organized as follows. In Sec. II, we introduce the model system and the theoretical method for the calculation of AMR in magnetic nanocontacts. In Sec. III, we calculate the AMR in the contact or ballistic regime (BAMR). In Sec. IV, we treat AMR in the coherent tunneling regime (TAMR), while in Sec. V, we treat AMR in the sequential tunneling regime. Finally, in Sec. VI, we conclude the paper summarizing the main results.

\section{MODEL AND METHODOLOGY}

As a model system for ferromagnetic nanocontacts, we consider two semi-infinite Ni 1D chains with lattice parameter $a$, separated by a gap $d>a$, as shown in Fig. 1. This model shares most of the relevant features with realistic nanocontact models, like, e.g., the low coordination of the tip atoms of the two electrodes and elastic electron scattering due to the gap. On the other hand, the one dimensionality and the resulting rotational invariance of our model considerably simplify the calculations of the SOC and the interpretation of the results. Such one-dimensional models have been employed before to study fundamental properties of atomicsized nanocontacts. ${ }^{27-29}$

We calculate the electronic structure of the system using a combination of density functional theory in the local spin density approximation (LSDA) and a Green's function technique to account for the fact that, when $d \neq a$, the system is not translationally invariant. We split the system into three regions, left $(L)$ and right $(R)$ electrodes, described as semiinfinite $\mathrm{Ni}$ chains and the central region $(C)$ containing the three innermost atoms of each electrode, as shown in Fig. 1.

The electronic structure of both the electrodes and the central region is described by using effective one-body Hamiltonians obtained from ab initio calculations, performed with CRYSTAL $03^{30}$ on the LSDA level, and using a localized atomic orbital minimal basis set. CRYSTAL03, which does not include SOC, yields spin-polarized solutions along an arbitrary axis with majority and minority electrons. The SOC term $\hat{H}_{\mathrm{SO}}=\lambda \vec{L} \cdot \vec{S}$ is added to the converged self-consistent LSDA Hamiltonian $\hat{H}_{\text {LSDA }}$,

$$
\hat{H}=\hat{H}_{\mathrm{LSDA}}+\hat{H}_{\mathrm{SO}} .
$$

This post-self-consistent approach ${ }^{14,31}$ is justified in the case of $\mathrm{Ni}$, for which the SOC is much smaller than the exchange splittings and the bandwidths. We take $\lambda=70 \mathrm{meV}$ for the Ni $3 d$ orbitals.

The Green's function of the central region is obtained by means of the so-called partitioning technique,

$$
\hat{G}_{C}(E)=\left[E-\hat{H}_{C}-\hat{\Sigma}_{L}(E)-\hat{\Sigma}_{R}(E)\right]^{-1},
$$

where $\hat{H}_{C}$ is the total Hamiltonian (including SOC) of the $C$ region and $\hat{\Sigma}_{L}$ and $\hat{\Sigma}_{R}$ are self-energies that take into account the coupling of the central region to the two electrodes. ${ }^{32,33}$

In the coherent regime, i.e., at low temperatures and small bias voltages when inelastic scattering events can be neglected, we use the Landauer formalism to calculate the conductance of the system which is obtained from the transmission function. The transmission function, in turn, can be calculated by means of the expression of Caroli et al. from the Green's function of the central region [Eq. (2)] and the so-called coupling matrices, $\hat{\Gamma}_{L}(E):=i\left(\hat{\Sigma}_{L}-\hat{\Sigma}_{L}^{\dagger}\right)$ and $\hat{\Gamma}_{R}(E)$ $:=i\left(\hat{\Sigma}_{R}-\hat{\Sigma}_{R}^{\dagger}\right)$, of the electrodes, ${ }^{34}$

$$
T(E)=\operatorname{Tr}\left[\hat{G}_{C}(E) \hat{\Gamma}_{L}(E) \hat{G}_{C}^{\dagger}(E) \hat{\Gamma}_{R}(E)\right] .
$$

The zero-bias conductance is then given by $G=\frac{e^{2}}{h} T\left(\epsilon_{F}\right)$. The orbital projected density of states $\rho_{\alpha}$ and the density of states of the central region $\rho$ can be calculated from the Green's function, $\quad \rho_{\alpha}(E)=-\frac{1}{\pi} \operatorname{Im}\left[G_{\alpha \alpha}(E)\right] \quad$ and $\quad \rho(E)=$ $-\frac{1}{\pi} \operatorname{Im} \operatorname{Tr}\left[\hat{G}_{C}(E)\right]$.

\section{CONTACT REGIME}

\section{A. Ideal chain}

Magnetic anisotropy comes from the combined action of both the crystal field that breaks the orbital rotational invariance and the atomic SOC term, which couples the spin polarization to the orbital degrees of freedom. The electronic structure of the ideal one-dimensional Ni chain $(d=a)$ presents a number of common features with $3 d$ and $4 d$ transition metals ${ }^{17,35}$ and permits us to understand the transport results for $d \neq a$. The bands close to the Fermi energy are formed by $s$ and $d$ orbitals. In the absence of SOC, rotational invariance around the chain axis permits us to classify the $d$ orbitals according to the projection of their angular momentum along the chain direction $m_{z}$. On top of that, a weak crystal field splits the otherwise degenerate $d$ levels into two doublets $E_{1}$ (linear combination of states with $\left.m_{z}= \pm 1\right)$ and $E_{2}\left(m_{z}\right.$ $= \pm 2)$ and a singlet $A_{1}\left(m_{z}=0\right)$, which is hybridized with the $s$ orbital. The orbital degeneracy of the doublets is kept by the bands of the chain, as long as SOC is not present. The bandwidth of the $E_{2}$ is significantly smaller than that of $E_{1}$ due to the smaller overlap of the $E_{2}$ orbitals. As a result, the $E_{2}$ bands yield a higher density of states.

The combined action of SOC and magnetism alters this situation. ${ }^{14,17}$ When the magnetization is pointing perpendicular to the chain axis $\left(\theta=90^{\circ}\right), \mathrm{SOC}$ acts as an effective magnetic field acting over $L_{x}$ that has to compete with the $L_{z}^{2}$-like terms arising from the crystal field, which happens to be dominant. As a result, the bands for $\theta=90^{\circ}$ look very similar to those without SOC, except in the points where bands with $m_{z} \sigma$ and $m_{z} \pm 1, \sigma \mp 1$ intersect, which are far away from $\epsilon_{F}$ in the case of Ni. Therefore, for $\theta=90^{\circ}$, the effect of SOC on transport is negligible. In contrast, when magnetization is pointing along the chain axis $\left(\theta=0^{\circ}\right)$, SOC shifts the bands by an amount $\lambda m_{z} \sigma$, where $m_{z}$ and $\sigma$ are the 

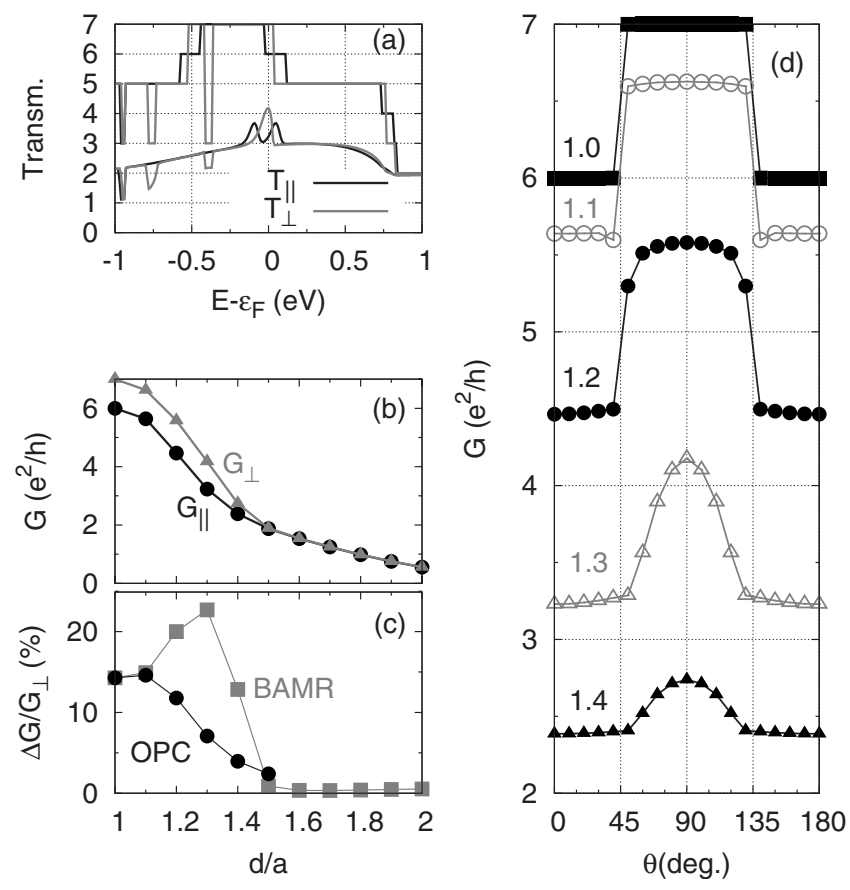

FIG. 2. (a) Transmission for an ideal infinite Ni chain $(d=a$, upper curves) and for two semi-infinite Ni chains separated by $d$ $=1.3 a$ (lower curves) for magnetization parallel (black) and perpendicular (gray) to the chain axis. (b) Zero-bias conductance $G$ as a function of $d$ for magnetization parallel (black) and perpendicular (gray) to the chain axis. (c) BAMR (gray boxes) and OPC (black circles) as a function of $d$. (d) $G$ as a function of $\theta$ for different values of $d / a$ : 1.0 (full black boxes), 1.1 (gray circles), 1.2 (black full circles), 1.3 (gray triangles), and 1.4 (black full triangles).

projection of the spin and orbital momentum along the chain. As a result, the $E_{2}$ and $E_{1}$ orbital doublets are split so that one of the two minority $E_{2}$ bands is shifted below the Fermi energy, compared to the $\theta=90^{\circ}$ case. This can be seen in the stepwise curves in Fig. 2(a), which correspond to $T_{\|}(E)$ $\equiv T\left(E, \theta=0^{\circ}\right)$ and $T_{\perp}(E) \equiv T\left(E, \theta=90^{\circ}\right)$ for the ideal chain. At the Fermi energy, $T_{\perp}(E) \neq T_{\|}(E)$. This change is responsible for BAMR, ${ }^{14}$ which is defined as BAMR $\equiv \frac{\Delta G}{G_{\perp}} \times 100$, where $\Delta G \equiv G_{\perp}-G_{\|}$.

The interplay between SOC and magnetization results in a nonzero orbital moment density along the magnetization direction. The largest orbital moment occurs when $\theta=0$, i.e., when the magnetization is along the chain. ${ }^{17}$ The orbital polarization current (OPC) defined as

$$
\mathrm{OPC} \equiv \frac{\sum_{m} T_{m}-T_{-m}}{T(E)},
$$

where $T_{m}$ is the transmission of the $d$ orbitals with $m= \pm 2$ or $m= \pm 1$ along the chain direction, vanishes when $\theta=90^{\circ}$ but is nonzero when $\theta=0$. Interestingly, there is a perfect one-toone correspondence between the OPC and the BAMR in the case of the ideal chain without scattering. It is also apparent that the existence of an orbital magnetic moment is not a sufficient condition for having a nonzero OPC, very much like spin polarization does not necessarily imply a spinpolarized current. ${ }^{23}$

\section{B. Effect of weak scattering}

Now, we see how elastic scattering, controlled with the chain separation $d$, affects BAMR. The stretched bond mimics the contact region. This perturbation preserves the axial symmetry of the ideal chain but introduces scattering. As a consequence, $T(E)$, shown in Fig. 2(a), is not quantized anymore, as expected, ${ }^{22,23}$ and yet the BAMR [Fig. 2(c)] is close to that of the ideal case for values of $d / a \leqslant 1$.4. Relatedly, the $G(\theta)$ curve is not stepwise (as is the case of the ideal chain) anymore when scattering is included [Fig. 2(d)]. On the other hand, the $G(\theta)$ curve is also different from bulk behavior where $G(\theta) \propto \cos ^{2} \theta$. The quantized step in the ideal case $(d=a)$ that corresponds to the critical angle at which the $E_{2}$ band is pushed below the Fermi energy ${ }^{14}$ becomes progressively smoother as the gap between the chains increases. Our $G(\theta)$ curves including scattering agree with those of the experiments. ${ }^{10}$ This is one of the important results of the model.

As $d$ increases, the scattering increases and $G$ goes down, but interestingly, the BAMR signal first increases slightly for $d / a \leqslant 1.3$ before finally going down with increasing scattering. The initial increase in BAMR is related to the initially stronger decrease in the contribution of the $A_{1}$ channel to the conductance. The $A_{1}$ channel is not affected by the SOC in contrast to the $E_{2}$ channels that are mainly responsible for the BAMR signal. The decrease in the BAMR signal for larger values of $d$ is expected within the framework of our model, since the relative contribution to the conductance of the $d$ channels compared to the $s$ channel decreases as the gap opens. The reason for this is the shorter spread of the $d$ orbitals compared to the $s$ orbitals. ${ }^{23}$ Relatedly, the OPC [Fig. 2(c)] also decreases as $d$ increases. Removing the contribution of the $s$ channel to the conductance would thus enhance BAMR. This could be accomplished, e.g., by oxidation of the contact. ${ }^{36}$

\section{COHERENT TUNNELING REGIME}

In this section, we study the anisotropic magnetoresistance in the regime of weakly coupled semi-infinite chains. In Figs. 3(a) and 3(c), we plot the Landauer transmission $T(E)$ (calculated from the expression of Caroli et al. [Eq. (3)] for $d=4 a$, definitely in the tunnel regime, and the density of states (DOS) projected onto the tip atom of a semi-infinite $\mathrm{Ni}$ chain both for $\theta=0^{\circ}\left[\rho_{\|}(E)\right]$ and $\theta=90^{\circ}\left[\rho_{\perp}(E)\right]$. The very small transmission is dominated by the $s$ channel and, therefore, quite independent of $\theta$. In contrast, the DOS is very different for $\theta=0^{\circ}$ and $\theta=90^{\circ}$. The two peak structure around $\epsilon_{F}$ for $\theta=0^{\circ}$ is related to the split $E_{2}$ bands, which merge when $\theta=90^{\circ}$. In Figs. 3(b) and 3(d), we plot the zerobias conductance $G(\theta)$ and the DOS at the Fermi level $\rho\left(\epsilon_{F}, \theta\right)$ as a function of $\theta$. Whereas the maximal change in the conductance is smaller than $1 \%$, the change in the DOS exceeds $200 \%$. This challenges the simplistic link between DOS and tunnel conductance.

In the tunneling regime, the Landauer formula can be rewritten as (see Appendix)

$$
G_{\text {tunnel }}=\frac{4 e^{2}}{h} \sum_{\alpha, \beta}\left|V_{\alpha \beta}\right|^{2} \rho_{\alpha}^{L}\left(\epsilon_{F}\right) \rho_{\beta}^{R}\left(\epsilon_{F}\right),
$$

where $V_{\alpha \beta}$ is the matrix element of the Hamiltonian connecting the $\alpha$ and $\beta$ atomic orbitals of the tip atoms of the two $\mathrm{Ni}$ 

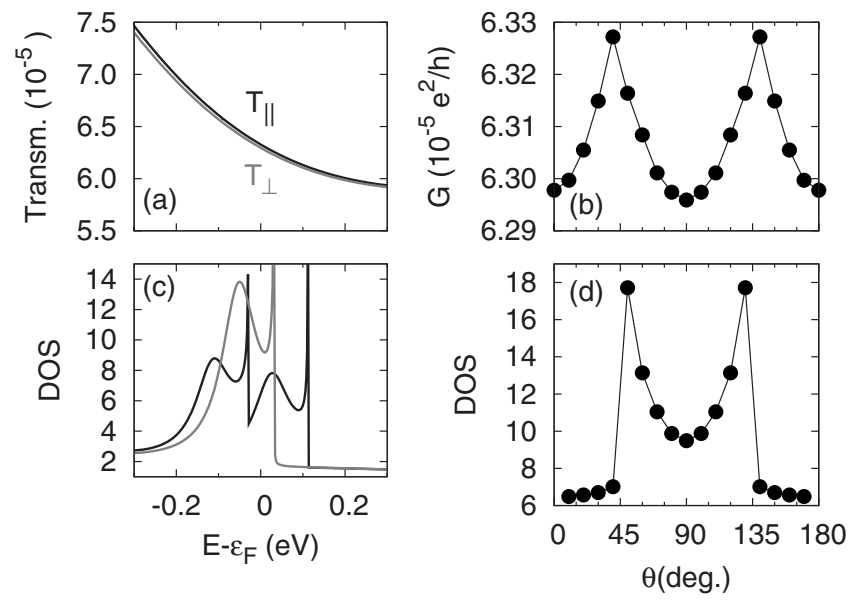

FIG. 3. Tunnel regime $(d=4 a)$ : (a) transmission function for magnetization angles $\theta=0^{\circ}$ (black) and $\theta=90^{\circ}$ (gray). (b) Zero-bias conductance in dependence of the magnetization angle $\theta$. (c) DOS projected onto tip atom as a function of energy for $\theta=0$ (black) and $\theta=90^{\circ}$ (gray). (d) DOS projected onto tip atom at the Fermi level as a function of $\theta$.

chains and $\rho_{\alpha}^{L, R}\left(\epsilon_{F}\right)$ is the orbital-resolved DOS at the Fermi energy, i.e., the DOS projected onto an atomic orbital $\alpha$ of a tip atom. By using this expression, the conductance calculated in Fig. 3(a) from the expression of Caroli et al. is indeed nicely reproduced. Note that the standard approximation by which the conductance is proportional to the product of the DOS of the tip atoms, $G \propto \Sigma_{\alpha} \rho_{\alpha}^{L}\left(\epsilon_{F}\right) \Sigma_{\alpha} \rho_{\beta}^{R}\left(\epsilon_{F}\right)$, is obtained only if the $V_{\alpha \beta}$ matrix is assumed to be proportional to the identity; i.e., the tunneling matrix elements are assumed to conserve the orbital index and to be equal in size. However, this is far from being the case when $d$ and $s$ orbitals are involved. In fact, in the case considered here, the conductance is completely dominated by the $V_{s, s}$ term, for which the orbitally resolved DOS $\rho_{s}^{L, R}$ is essentially independent of $\theta$. As a result, the strong dependence of the global density of states on $\theta$ is not followed, in this case, by a strong dependence of the conductance on $\theta$. Notice that since the transmission is dominated by the $s$ channel, both the orbital polarization of the current and the AMR are negligible. In general, an anisotropy in the DOS is not a sufficient condition to have AMR.

The small variation of $G(\theta)$ in Fig. 3(b) can be traced back to the variation of $\epsilon_{F}$ as a function of $\theta$ and the nonflat $\rho_{s}^{L, R}(E)$. In Fig. 4(a), we plot $\epsilon_{F}(\theta)$ for a semi-infinite $\mathrm{Ni}$ chain. $\Delta \epsilon_{F} \equiv \epsilon_{F}\left(0^{\circ}\right)-\epsilon_{F}\left(90^{\circ}\right)$ can be as large as $10 \mathrm{meV}$. This change leads naturally to the second scenario for enhancement of the AMR, considered in the next section: in a situation of resonant transport through the change of the chemical potential, $\boldsymbol{\epsilon}_{F}$ as a function of the magnetization direction can result in a large variation of $G$, regardless of the degree of orbital polarization of the current. It has been recently suggested that these resonances could arise as localized tip states in $\mathrm{Ni}$ wires thicker than those considered here. $^{26}$

\section{SEQUENTIAL TUNNELING REGIME}

In this section, we consider a different scenario, motivated by recent experiments ${ }^{15}$ and by the remarks at the end of the
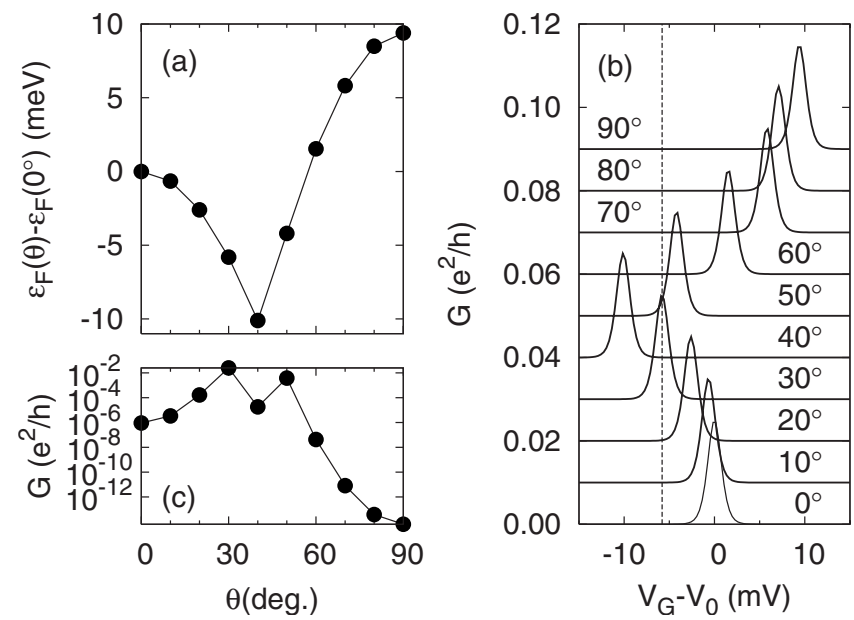

FIG. 4. (a) Variation of the Fermi energy of the Ni chain as a function of $\theta$. (b) $G\left(V_{G}, \theta\right)$ for a SET device coupled to the Ni chains. The curves are vertically shifted. (c) $G(5 \mathrm{meV}, \theta)$ in logarithmic scale.

previous section. We study a single electron transistor (SET) with $\mathrm{Ni}$ electrodes ${ }^{19,37,38}$ and a nonmagnetic central island (CI) with a discrete electronic spectrum. The CI is weakly coupled to the electrodes, so that the levels acquire a broadening $\Gamma$. The position of these levels can be electrically tuned with a gate. Whenever a level of the CI is in resonance with the Fermi energy, the zero-bias conductance of the system has a maximum. We assume that both the level spacing of the CI states $\Delta E$ and the charging energy $E_{Q}$ are much larger than the temperature $k_{B} T$ which is larger than $\Gamma$. Under these conditions, the system is in the Coulomb blockade regime.

In equilibrium, the chemical potential of the central island and that of the electrodes must be the same: ${ }^{24} \epsilon_{F}(\theta)=E_{C}$ $+\epsilon_{N}+e V_{G}$, where $N$ is the number of electrons in the CI that satisfies this condition and $\epsilon_{N}$ is the energy level occupied by the last electron. From this equation, we immediately see that the charge state of the central island can be controlled both with the gate and with the orientation of the magnetization of the electrodes. ${ }^{15,25}$ This effect is reminiscent of the so-called magneto-Coulomb effect, in which the chemical potential of the electrode is varied with the intensity of the applied field. ${ }^{39}$ Here, the chemical potential is changed by rotating the applied field.

In the $E_{Q}>k T>\Gamma$ situation, the linear conductance of the SET can be obtained by using either the finite temperature Landauer approach ${ }^{33}$ or the sequential transport theory, ${ }^{24}$

$$
G=\frac{e^{2}}{h} \frac{\Gamma}{8 k_{B} T} \cosh ^{-2}\left(\Delta / 2 k_{B} T\right),
$$

where $\Delta=E_{N}\left(V_{G}\right)+\frac{e^{2}}{2 C}-\epsilon_{F}(\theta)$. In Fig. 4(b), we plot $G\left(V_{G}, \theta\right)$ for a SET with Ni electrodes. We take $k T=5 \Gamma=0.5 \mathrm{meV}$. The gate is chosen so that for $\theta=0$ the conductance is maximal. As $\theta$ changes, the chemical potential of the electrodes moves away from the peak. In Fig. 4(c), we plot $G(\theta)$ for $V_{G}$ corresponding to the vertical line in Fig. 4(b). Notice the logarithmic scale and the huge AMR, which might have practical applications. Notice that crossing the conductance 
peak, either by gate application or magnetization rotation, implies charging the CI by one electron. ${ }^{15,25}$ The results of Fig. 4(b) assume that $\Gamma$ is independent of $\theta$, which is true as long as the resonant level is not coupled to the $E_{2}$ and $E_{1}$ bands. The height of the $G\left(V_{G}\right)$ curves would depend on $\theta$ otherwise. In principle, a complete characterization of the $G\left(V_{G}, \theta\right)$ curve would yield the $\epsilon_{F}(\theta)$ and $\Gamma(\theta)$ functions, which would provide valuable information of the electronic structure of the electrodes.

\section{SUMMARY AND CONCLUSIONS}

We have presented $a b$ initio quantum transport calculations of Ni nanocontacts as a function of the magnetization direction $\theta$ going from the ballistic to the tunnel regime. We have shown that AMR is unrelated from quantization of conductance, which is an artifact of the scattering-free calculations and not expected in transition metal nanocontacts. We also show that a large variation of the density of states at $\epsilon_{F}$ as a function of $\theta$ is not a sufficient condition for large AMR. We identify two sufficient conditions to obtain largely enhanced AMR in quantum transport. First, in the coherent regime (contact and tunneling), large AMR is related to a large degree of orbital polarization of the current for a selected direction of the magnetization. Second, in systems with resonances close to $\epsilon_{F}$, as it happens in single electron transistors with ferromagnetic electrodes, large AMR is related to a large variation of the chemical potential $\epsilon_{F}$ of the electrode as a function of $\theta$. We report an ab initio calculation for this quantity. These findings shed light on the choice of materials and the design of nanostructures with enhanced anisotropic magnetoresistance.

\section{ACKNOWLEDGMENTS}

We acknowledge R. Aguado, L. Brey, E. Tsymbal, E. Tosatti, and C. Untiedt for useful discussions. We acknowledge Spanish MEC and Generalitat Valenciana for funding grants (MAT2007-65487), Ramon y Cajal Program (GVACCOMP07/054), and Consolider (CSD2007-0010).

\section{APPENDIX A: DERIVATION OF TUNNELING FORMULA}

For completeness, we derive Eq. (5) from the Landauer formalism in the limit of weak coupling between the electrodes. Equation (5) can also be obtained from the Kubo formula (see, e.g., the book by Mahan ${ }^{40}$ Sec. 9.3). Derivations similar to ours can be found in the literature..$^{33,41}$

We consider two semi-infinite electrodes $L$ and $R$ with atomically sharp tips separated by a distance $d$, as shown in Fig. 1. We label the tip atoms of the left and right leads 0 and 1 , respectively. Now the Green's function projected onto tip atom 0 is given by

$$
\hat{G}_{0}(E)=\left[E-\hat{H}_{0}-\hat{\Sigma}_{L}(E)-\hat{\Sigma}_{R}(E)\right]^{-1},
$$

where $\hat{\Sigma}_{L}$ is the self-energy representing the rest of the left electrode without tip atom 0 , while $\hat{\Sigma}_{R}$ presents the self- energy of the entire right electrode including the tip atom 1. Thus, the right self-energy can be expressed by the Green's function of the isolated right electrode $\hat{g}_{1}^{R}$ and the coupling $\hat{V}$ between the left and the right tip atoms as

$$
\hat{\Sigma}_{R}=\hat{V} \hat{g}_{1}^{R} \hat{V}^{\dagger} .
$$

In the tunneling regime, i.e., for $d \gg a$, when the coupling $\hat{V}$ becomes very weak, the contribution of the right self-energy to $\hat{G}_{0}$ can be neglected, so that $\hat{G}_{0}$ becomes equal to the Green's function of the isolated left lead projected onto the tip atom $\hat{g}_{0}^{L}$,

$$
\hat{G}_{0}(E) \approx\left[E-\hat{H}_{0}-\hat{\Sigma}_{L}(E)\right]^{-1} \equiv \hat{g}_{0}^{L}(E) .
$$

The expression of Caroli et al. ${ }^{34}$ for the Landauer transmission through the tip atom thus becomes

$$
T(E) \approx \operatorname{Tr}\left[\hat{g}_{0}^{L}(E) \hat{\Gamma}_{L}(E)\left(\hat{g}_{0}^{L}\right)^{\dagger}(E) \hat{\Gamma}_{R}(E)\right] .
$$

The coupling matrix of the right lead $\hat{\Gamma}_{R}$ can be rewritten in terms of the spectral function of the isolated right lead projected onto the tip atom, $\hat{a}_{1}^{R}:=i\left[\hat{g}_{1}^{R}-\left(\hat{g}_{1}^{R}\right)^{\dagger}\right]$, as

$$
\hat{\Gamma}_{R}:=i\left(\hat{\Sigma}_{R}-\hat{\Sigma}_{R}^{\dagger}\right)=\hat{V} \hat{a}_{1}^{R} \hat{V}^{\dagger}
$$

The first three terms in Eq. (A4) are computed by using the algebraic identity $\hat{g}_{0}^{L} \hat{\Gamma}_{L}\left(\hat{g}_{0}^{L}\right)^{\dagger}=i\left[\hat{g}_{0}^{L}-\left(\hat{g}_{0}^{L}\right)^{\dagger}\right]=\hat{a}_{0}^{L}$, where $\hat{a}_{0}^{L}$ is the spectral function of the isolated left lead projected onto the tip atom 0 , where we find for the transmission in the tunneling regime,

$$
T(E) \approx \operatorname{Tr}\left[\hat{a}_{0}^{L}(E) \Gamma_{R}(E)\right]=\operatorname{Tr}\left[\hat{a}_{0}^{L}(E) \hat{V} \hat{a}_{1}^{R}(E) \hat{V}^{\dagger}\right] .
$$

Thus, the zero-bias conductance, which is given by the transmission function at the Fermi energy, can be approximated in the tunneling regime by

$$
\begin{aligned}
G & =\frac{e^{2}}{h} T\left(\epsilon_{F}\right) \approx \frac{e^{2}}{h} \operatorname{Tr}\left[\hat{a}_{0}^{L}\left(\epsilon_{F}\right) \hat{V} \hat{a}_{1}^{R}\left(\epsilon_{F}\right) \hat{V}^{\dagger}\right] \\
& =\frac{e^{2}}{h} \sum_{\alpha, \alpha^{\prime}, \beta, \beta^{\prime}} a_{\alpha \alpha^{\prime}}^{L}\left(\epsilon_{F}\right) V_{\alpha^{\prime} \beta} a_{\beta \beta^{\prime}}^{R}\left(\epsilon_{F}\right) V_{\alpha \beta^{\prime}}^{*},
\end{aligned}
$$

where in the last step, we have labeled the states on the left tip by $\alpha$ and $\alpha^{\prime}$ and states on the right tip by $\beta$ and $\beta^{\prime}$. The spectral functions $\hat{a}^{L}$ and $\hat{a}^{R}$ are diagonal in the basis of eigenstates of the isolated left and right leads, and the diagonal elements yield the DOS projected onto the eigenstates, $a_{\alpha \alpha}^{L}=2 \rho_{\alpha}^{L}$ and $a_{\beta \beta}^{R}=2 \rho_{\beta}^{R}$, where $\alpha$ and $\beta$ now label the projections of the eigenstates onto the tip atoms. Thus, we obtain Eq. (5),

$$
G \approx \frac{e^{2}}{h} \sum_{\alpha, \beta} \rho_{\alpha}^{L}\left(\epsilon_{F}\right) V_{\alpha \beta} \rho_{\beta}^{R}\left(\epsilon_{F}\right) V_{\alpha \beta}^{*} .
$$

Notice that this result relates the tunnel conductance to the product of the orbital-resolved DOS of the electrodes, as opposed to the total DOS. 
${ }^{1}$ P. Gambardella, S. Rusponi, M. Veronese, S. S. Dhesi, C. Grazioli, A. Dallmeyer, I. Cabria, R. Zeller, P. H. Dederichs, K. Kern, C. Carbone, and H. Brune, Science 300, 1130 (2003).

${ }^{2}$ T. O. Strandberg, C. M. Canali, and A. H. MacDonald, Nat. Mater. 6, 648 (2007).

${ }^{3}$ C. Gould, C. Rüster, T. Jungwirth, E. Girgis, G. M. Schott, R. Giraud, K. Brunner, G. Schmidt, and L. W. Molenkamp, Phys. Rev. Lett. 93, 117203 (2004).

${ }^{4}$ C. Rüster, C. Gould, T. Jungwirth, J. Sinova, G. M. Schott, R. Giraud, K. Brunner, G. Schmidt, and L. W. Molenkamp, Phys. Rev. Lett. 94, 027203 (2005).

${ }^{5}$ A. D. Giddings, M. N. Khalid, T. Jungwirth, J. Wunderlich, S. Yasin, R. P. Campion, K. W. Edmonds, J. Sinova, K. Ito, K.-Y. Wang, D. Williams, B. L. Gallagher, and C. T. Foxon, Phys. Rev. Lett. 94, 127202 (2005).

${ }^{6}$ H. Saito, S. Yuasa, and K. Ando, Phys. Rev. Lett. 95, 086604 (2005).

${ }^{7}$ J. Moser, A. Matos-Abiague, D. Schuh, W. Wegscheider, J. Fabian, and D. Weiss, Phys. Rev. Lett. 99, 056601 (2007).

${ }^{8}$ A. N. Grigorenko, K. S. Novoselov, and D. J. Maps, arXiv:condmat/0611751 (unpublished).

${ }^{9}$ K. I. Bolotin, F. Kuemmeth, and D. C. Ralph, Phys. Rev. Lett. 97, 127202 (2006).

${ }^{10}$ M. Viret, M. Gabureac, F. Ott, C. Fermon, C. Barreteau, G. Autes, and R. Guirado-Lopez, Eur. Phys. J. B 51, 1 (2006).

${ }^{11}$ Z. K. Keane, L. H. Yu, and D. Natelson, Appl. Phys. Lett. 88, 062514 (2006).

${ }^{12}$ A. Sokolov, C. Zhang, E. Y. Tsymbal, J. Redepenning, and B. Doudin, Nat. Nanotechnol. 2, 171 (2007).

${ }^{13}$ Whereas in the context of mesoscopics the term ballistic refers to lack of any scattering (Ref. 33), in the context of nanoconstrictions, the term ballistic refers to the lack of inelastic scattering only and is thus synonymous with elastic or coherent.

${ }^{14}$ J. Velev, R. F. Sabirianov, S. S. Jaswal, and E. Y. Tsymbal, Phys. Rev. Lett. 94, 127203 (2005).

${ }^{15}$ J. Wunderlich, T. Jungwirth, B. Kaestner, A. C. Irvine, A. B. Shick, N. Stone, K.-Y. Wang, U. Rana, A. D. Giddings, C. T. Foxon, R. P. Campion, D. A. Williams, and B. L. Gallagher, Phys. Rev. Lett. 97, 077201 (2006).

${ }^{16}$ J. Fernández-Rossier, D. Jacob, C. Untiedt, and J. J. Palacios, Phys. Rev. B 72, 224418 (2005).

${ }^{17}$ Y. Mokrousov, G. Bihlmayer, S. Heinze, and S. Blugel, Phys. Rev. Lett. 96, 147201 (2006).

${ }^{18}$ K. Bolotin, F. Kuemmeth, A. N. Pasupathy, and D. C. Ralph, Nano Lett. 6, 223 (2006).

${ }^{19}$ A. N. Pasupathy, R. C. Bialczak, J. Martinek, J. E. Grose, L. A. K. Donev, P. L. McEuen, and D. C. Ralph, Science 306, 86
(2004).

${ }^{20}$ L. Brey, C. Tejedor, and J. Fernández-Rossier, Appl. Phys. Lett. 85, 1996 (2004); P. Sankowski, P. Kacman, J. A. Majewski, and T. Dietl, Phys. Rev. B 75, 045306 (2007); A. Matos-Abiague and J. Fabian, arXiv:cond-mat/0702387 (unpublished).

${ }^{21}$ E. Scheer, N. Agraï, J. C. Cuevas, A. Levy Yeyati, B. Ludoph, A. Martín-Rodero, G. R. Bollinger, J. M. van Ruitenbeek, and C. Urbina, Nature (London) 394, 154 (1998).

${ }^{22}$ C. Untiedt, D. M. T. Dekker, D. Djukic, and J. M. van Ruitenbeek, Phys. Rev. B 69, 081401(R) (2004).

${ }^{23}$ D. Jacob, J. Fernández-Rossier, and J. J. Palacios, Phys. Rev. B 71, 220403(R) (2005); D. Jacob and J. J. Palacios, ibid. 73, 075429 (2006).

${ }^{24}$ C. W. J. Beenakker, Phys. Rev. B 44, 1646 (1991).

${ }^{25}$ J. Fernández-Rossier, R. Aguado, and L. Brey, Phys. Status Solidi C 3, 4231 (2006).

${ }^{26}$ J. D. Burton, R. F. Sabirianov, J. P. Velev, O. N. Myrasov, and E. Y. Tsymbal, arXiv:cond-mat/0703345 (unpublished).

${ }^{27}$ A. Delin and E. Tosatti, Surf. Sci. 566-568, 262 (2004).

${ }^{28}$ A. Smogunov, A. Dal Corso, and E. Tosatti, Surf. Sci. 566-568, 390 (2004).

${ }^{29}$ A. Dal Corso, A. Smogunov, and E. Tosatti, Phys. Rev. B 74, 045429 (2006).

${ }^{30}$ Theoretical Chemistry Group, CRYSTAL03, 1.0.2, Release Universita' Di Torino, Torino, Italy.

${ }^{31}$ L. Fernández-Seivane, M. A. Oliveira, S. Sanvito, and J. Ferrer, J. Phys.: Condens. Matter 18, 7999 (2006).

${ }^{32}$ D. Jacob, Ph.D. thesis, Universidad de Alicante, 2007.

${ }^{33}$ S. Datta, Electronic Transport in Mesoscopic Systems (Cambridge University Press, Cambridge, 1995).

${ }^{34}$ C. Caroli, R. Combescot, P. Nozieres, and D. Saint-James, J. Phys. C 4, 916 (1971).

${ }^{35}$ M. Wierzbowska, A. Delin, and E. Tosatti, Phys. Rev. B 72, 035439 (2005).

${ }^{36}$ D. Jacob, J. Fernández-Rossier, and J. J. Palacios, Phys. Rev. B 74, 081402(R) (2006).

${ }^{37}$ R. S. Liu, H. Pettersson, L. Michalak, C. M. Canali, and L. Samuelson, Nano Lett. 7, 81 (2007).

${ }^{38}$ P. Seneor, A. Bernarnd-Mantel, and F. Petroff, J. Phys.: Condens. Matter 19, 165222 (2007).

${ }^{39}$ K. Ono, H. Shimada, and Y. Ootuka, J. Phys. Soc. Jpn. 66, 1261 (1997).

${ }^{40}$ G. D. Mahan, Many-Particle Physics 2nd ed. (Plenum, New York, 1990).

${ }^{41}$ S. Maekawa and T. Shinjo, Spin Dependent Transport in Magnetic Nanostructures (CRC, Boca Raton, 2002). 